\title{
La auditoría en la gestión de la transparencia del sector público
}

\section{Audit in the management of public sector transparency}

\author{
Vicent Giménez-Chornet \\ vigicho@har.upv.es \\ Universitat Politècnica de València
}

\begin{abstract}
Resumen
La transparencia en el sector público cubre muchos ámbitos, además de la propia administración pública. La normativa española ha regulado recientemente, tanto a nivel estatal como de los gobiernos autonómicos, los criterios para implementar un gobierno transparente que garantice la confianza de los ciudadanos con sus gobernantes. Se analiza si el sistema de la gestión de la transparencia ha asegurado una independencia frente al poder ejecutivo, y si ese sistema permite realizar auditorías confiables, especialmente independientes, que inspeccionen el grado de cumplimiento de la transparencia en el sector público.
\end{abstract}

\section{Palabras clave}

Transparencia; Auditorías; Sector público; Gobierno confiable; Acceso a la información

\begin{abstract}
Transparency in the public sector covers many areas, in addition to the public administration itself. Spanish legislation has recently regulated, both at the state level and the regional governments, the criteria for implementing a transparent government that guarantees the confidence of citizens with their governments. It is analyzed if the transparency management system has ensured an independence from the executive power, and if that system allows to perform reliable, especially independent, audits, which inspects the degree of compliance with transparency in the public sector.
\end{abstract}

\section{Keywords}

Transparency; Audits; Public sector; Reliable government; Access to information 
Recibido: 7/12/2017

Aceptado: 15/12/2017

DOI: http://dx.doi.org/10.5557/IIMEI8-N15-111123

Descripción propuesta: Giménez-Chornet, Vicent, 2017. La auditoría en la gestión de la transparencia del sector público. Métodos de información, 8(15), pp. 111-123.

\section{Importancia de la transparencia en el sector público}

La transparencia se puede interpretar desde varios puntos de vista. Las funciones del sector público son amplias, y muchas de ellas deben estar sometidas a un ejercicio de la transparencia en la comunidad a la que sirven.

La transparencia puede estar en el ámbito político (representantes parlamentarios): queremos políticos transparentes y exigiremos saber de sus actos, el origen y los objetivos de sus decisiones políticas, lo que nos cuesta a los ciudadanos su ejercicio, la coherencia deontológica entre su programa político y sus actuaciones públicas, ya que, al fin y al cabo, los hemos elegido como nuestros representantes, y les pagamos su sueldo con nuestros impuestos. La transparencia también se puede ver desde los medios de comunicación. Los periodistas realizan una labor extraordinaria en la búsqueda de información para comunicarla a los ciudadanos y cumplir con su misión de informar para que la toma de decisiones de los ciudadanos se dé con los argumentos pertinentes. Dado que no existe la objetividad en el ejercicio del periodismo, lo que no es en absoluto una connotación negativa, los ciudadanos les podemos exigir transparencia en su actividad, es decir, si acceden a la mayor cantidad de fuentes de información para dar la noticia en su globalidad, si practican la constatación de la veracidad de las informaciones, si realizan periodismo de investigación o se limitan a las "notas de prensa" (Carriedo 2007), si existen grupos de presión a la hora de informar, detectables por el sistema de financiación, etc. Es sabida la gran influencia de los medios de comunicación en la generación de conocimiento en la ciudadanía, con todas las subjetividades e intencionalidades de ese conocimiento, y es sabido también su papel social de ejercer el derecho a saber sobre cualquier actividad de la comunidad: políticos y política, empresas, salud, etc. 
En el ámbito del sistema democrático, la e-democracia o la democracia participativa, también se utiliza el término transparencia, relacionándolo con la implantación de mecanismos para la participación directa de los ciudadanos en la gestión de la comunidad, en el control de los gobernantes mediante su participación directa o mediante la elección de representantes fiscalizadores o de control de los organismos públicos, para garantizar la independencia de estos órganos de control respecto al Poder Ejecutivo. El objetivo es, generalmente, desvincular a los propios gobernantes de la elección del personal que ocupa órganos de control de su propia administración. Alcanzar la transparencia en la democracia pasa por realizar acciones concretas para fomentar que los ciudadanos ejerzan el control de las instituciones u organismos públicos, como una de las medidas para combatir, entre otras, la corrupción o la deficiente gestión de los órganos del Estado.

La transparencia también está relacionada con el derecho de acceso a la información que han generado las instituciones o las personas en el desarrollo de sus actividades en el sector público. Este aspecto se debe ver concretado en algunas acciones que garanticen el acceso a dicha información. Disponemos de una variada normativa jurídica que ha regulado a día de hoy dicho derecho al acceso, pero desconocemos si se cumple con eficiencia, si los ciudadanos pueden consultar el potencial de información que obra en el sector público catalogado como libre de restricciones legales para los ciudadanos. Así definimos "la transparencia en la gestión pública como la posibilidad de que los ciudadanos consigan acceder a cualquier tipo de información, registrada en cualquier soporte, generada por las entidades en el desarrollo de sus funciones" (Giménez-Chornet 2012). Hacemos hincapié en el concepto "posibilidad", que implica un desarrollo de acciones y gestión que los trasformen en "realidad", que los documentos sean realmente accesibles, mediante cualquier medio, para ello deben estar adecuadamente gestionados. Las diversas entidades públicas generan grandes cantidades de documentación (solamente cabe comprobarlo visitando los fondos de los archivos), generalmente en papel, y poco a poco va creciendo la documentación nacida digitalmente. Toda aquella documentación, en papel o electrónica, es imposible ponerla al servicio de los ciudadanos en Internet (eso es una utopía, pues haría falta una masa ingente de personal describiendo y digitalizando la documentación, y unos servidores que soportasen un volumen de tráfico en Internet fuera del alcance presupuestario actual), por ello posibilitar el acceso a esa documentación e información pasa 
por dejar consultarla a los ciudadanos en los centros productores (la normativa jurídica lo tiene que hacer posible, y los gestores deben tener identificada y descrita dicha información mediante bases de datos, que se puedan difundir, sin grandes costes presupuestarios, en la red ), y escasamente alguna poca documentación se puede difundir digitalizada por Internet. Que cierta información o documentación se difunda por Internet no la consideramos un acto total de transparencia del sector público, dado que esto pasa por una selección de la información (siempre subjetiva), muy limitada a lo que realmente producen las entidades públicas. Esa difusión por Internet es, simplemente, un acto de difusión de información, positivo por el propio hecho en sí, pero no un ejercicio de transparencia completa de las entidades públicas en un país.

El creciente interés por los Open Data va ligado, por una parte, a que la sociedad disponga de una información beneficiosa para la investigación y, consecuentemente, a una mejora de la política pública y de la sociedad (Sá 2016), y por otra parte a la implantación de una política de transparencia en el ejercicio del gobierno público (Gunnlaugsdottir 2016), unida ésta a un código ético para la práctica de dicha transparencia (Oztoprak 2016). La tecnología (TIC) permite que avancemos en establecer unas relaciones próximas e inmediatas entre gobiernos y ciudadanos. A esta tecnología se le ha llamado recientemente Tecnología Cívica, que hace posible la participación de los ciudadanos de los datos abiertos del gobierno (McNutt 2016). Recientemente los estados van poniendo a la disposición de los ciudadanos grandes cantidades de conjuntos de datos en abierto que abarcan amplios campos de las actividades desarrolladas, como son la educación, la sanidad, la cultura, la economía o la población, etc. El gran reto es conseguir que esos datos sean usados por el público, propiciando la reutilización para tratar de crear nuevas oportunidades económicas (Okamoto 2016).

La disponibilidad y accesibilidad al conjunto de datos, al igual que a una amplia disposición de artículos académicos en abierto, supone un reto en cuanto a asegurar su acceso mantenido, que pueda mejorar con el tiempo, requiriendo un notable esfuerzo en recursos humanos, dinero, inversión de tiempo y protección de la propiedad intelectual. La innovación principal de la web debería permitir que las personas pudiesen enlazar documentos a nivel mundial, trabajando en los datos enlazados (Linked Data), y para ello es necesario también que se implemente una vinculación de las tecnologías (Linking Technologies) que 
permita la construcción de una colección de datos a partir de una variedad de centros suministradores de datos (Mayernik 2016).

La Unión Europea está desarrollando una política de datos abiertos con el objetivo de generar valor a través de la reutilización de la información disponible en el sector público, y esta política debe aplicarse de conformidad con los principios de la Directiva de Protección de Datos (Van Loenen 2016). A pesar de los esfuerzos de los estados de la Unión Europea en implantar la cultura de los datos abiertos que posibilite su reutilización, un estudio del portal español pone de manifiesto el bajo uso de la población de dichos datos, tal vez porque una parte de los ciudadanos no creen que las instituciones públicas ofrezcan suficiente información, o que el gobierno ofrezca aplicaciones fáciles para poder analizar e interpretar los datos (Gértrudix 2016). Uno de los factores que también puede afectar negativamente a la participación ciudadana en la reutilización de los datos abiertos de los gobiernos es la falta de control de calidad, que puede afectar a los metadatos, a suministrar datos incompletos, o a la falta de información sobre actualización de los datos (Vetrò 2016).

Los datos abiertos dan acceso a una información gubernamental que puede permitir a los ciudadanos controlar la calidad de los servicios públicos y cómo usan los gobiernos los recursos públicos. El estudio de Geginat (2016) demuestra que los países que han implementado medidas de transparencia y de buen gobierno son también países en los que las empresas no se ven obligadas a ponerse en contacto con un funcionario para obtener información básica, porque se encuentra en línea, indicando como un mayor acceso a la información se asocia con más democracia y menos corrupción, habiendo una relación clara con que la regulación empresarial es de mayor calidad en aquellos países en que la normativa jurídica es fácilmente accesible. Ya se han identificado carencias en cuanto que los datos abiertos de ciertos portales no poseen elementos estructurales y organizativos suficientes para apoyar a los ciudadanos en el acceso a la información gubernamental para la rendición de las cuentas públicas (Lourenço 2015). La indexación es uno de los grandes retos para una pertinente recuperación de la información, para localizar rápidamente los datos en las grandes cantidades de conjuntos de datos, siendo considerado por algunos autores que aún están en su etapa infantil en los sistemas de almacenamiento a pesar de su utilidad (Yaqoob 2016).

El objetivo del presente artículo, además de remarcar la importancia que tiene la transparencia en el sector público, es analizar en qué ámbitos una auditoría 
independiente puede generar confianza en la comunidad donde se ha implementado un sistema de transparencia, y examinar qué contempla el marco jurídico español en relación a una fiscalización o auditoría de sus actividades.

\section{Auditoría independiente de la transparencia}

La auditoría independiente del sector público es el instrumento que mejor puede garantizar la lucha contra la corrupción administrativa y la deficiente práctica de los gestores y de los responsables políticos. La auditoría independiente puede vigilar la aplicación estricta de la normativa jurídica. Existen numerosos estudios de carácter normativo sobre la transparencia y la rendición de cuentas, pero hacen falta planteamientos que ofrezcan unos resultados empíricos a gran escala sobre los instrumentos de la transparencia a nivel de los gobiernos y los políticos públicos. Se ha resaltado la importancia de los comités de auditoría en el sector privado como medio de restaurar la confianza de los inversores en los estados financieros de las empresas (Wang 2016), al igual que las auditorias en el sector público (Franklin 2007), pero la novedad, con las recientes leyes de transparencia del gobierno español y de las comunidades autónomas, es uno de los motivos de que no se haya abordado de forma sistémica la auditoría en la gestión y las acciones de la transparencia en el sector público, más allá de las obligaciones legales de control del propio sector público. La falta de una independencia en el sistema de auditoría perjudica a la rendición de cuentas, y en lo que respecta a la gestión de la transparencia la carencia de un modelo de auditoría independiente que aborde todas las acciones y responsabilidades del sector público incrementa el desarraigo de la comunidad, la desconfianza en el sector público y un ascenso del populismo político totalitario.

La organización Transparencia Internacional se autodenomina "la única organización no gubernamental a escala universal dedicada a combatir la corrupción, congregando a la sociedad civil, sector privado y los gobiernos en una amplia coalición global" (Transparencia Internacional, 2017), pero realmente se limita a enviar y rellenar un cuestionario por administraciones (a los ayuntamientos, a las comunidades autónomas, a los parlamentos, y a algunas entidades) para obtener evidencias sobre qué información han puesto disponible en la web. Más que transparencia se trata de una información al servicio del ciudadano en Internet que no corresponde a un sistema garantista 
de acceso a la información que genera y custodia el sector público, dando resultados que hacen presuponer que la transparencia activa (información en la web) es transparencia, y una administración pública con un resultado 100\% se le cataloga como totalmente transparente. Una auditoría podría demostrar, independientemente de la información que la corporación ha decidido plasmar en la web, el grado de cumplimiento de la normativa jurídica, y la accesibilidad real de los ciudadanos a la información del sector público.

\section{3. Ámbitos para una auditoría del sector público}

Existen diversos ámbitos en el sector público que están produciendo documentos susceptibles de auditoría como ejercicio de la transparencia. Los proyectos de auditoría deben identificar estos sectores, para poder evaluar los logros alcanzados y o para poder detectar las deficiencias. Ello permitirá a las distintas administraciones públicas y entidades vinculadas disponer de una radiografía de la situación y alcance de la transparencia en un momento dado.

Los distintos sectores en que se debe abordar el modelo de auditoria son:

A. Portales de transparencia

Las comunidades autónomas, al igual que el gobierno español o algunas administraciones locales, ha establecido un sitio web donde ofrecen a los ciudadanos de su comunidad información de diversa índole sobre sus actividades de gestión, especialmente la relacionada con los gastos derivados de las actividades de su administración.

\section{B. Data set}

Los data set, o conjunto de datos, es otro de los medios utilizados por las administraciones para que, en el ejercicio de su transparencia y de facilitar la reutilización de la información. Los repositorios de los data set se ponen a disposición de la comunidad en sitios web identificados como datos abiertos u Open Data.

C. Parlamentarismo y partidos políticos

Tanto la información generada en los parlamentos autonómicos como la generada en los partidos políticos, o por los mismos políticos, debe ser accesible 
a la sociedad: El ejercicio de la transparencia en la actividad política es una de las vías para acercar los partidos y el poder legislativo a la ciudadanía.

D. Entidades de derecho público y entidades privadas con subvenciones públicas

Las leyes españolas de transparencia (tanto la estatal como las de las comunidades autónomas) contemplan la obligación de ejercerla tanto a las entidades de derecho público como a las entidades privadas que dispongan de una cierta cantidad de subvención de fondos públicos. Estas entidades deben disponer de medios y servicios para ejercer la transparencia.

\section{E. Documentación pública accesible}

Con el término documentación pública accesible nos referimos a todos aquellos documentos de cualquier característica y soporte que se generan en las administraciones públicas pero que no son accesibles vía web. Nos referimos al ejercicio de la transparencia pasiva, ya que la documentación que genera el sector público es inmensamente mayor que la que está disponible en la web en los diferentes portales mencionados anteriormente. La transparencia pasiva tiene como objetivo, mediante un procedimiento regulado, poner a disposición del ciudadano cualquier tipo de documento o información no restringida por la legislación vigente, que solicite por vía reglada. Con ello se permite cumplir con el principio de acceso a la información pública de las administraciones.

\section{Marco jurídico de la transparencia en España: ausencia de auditorías}

La Ley 19/2013, de 9 de diciembre, de transparencia, acceso a la información pública y buen gobierno (España 2013), marca el inicio legislativo de la transparencia en España. Las leyes que posteriormente promulgarán las comunidades autónomas no podrán articular preceptos que contradigan dicha ley, que nació con una polémica sobre sus limitaciones en el ámbito de la transparencia, y especialmente sobre la cuestión de la independencia efectiva del órgano creado en la propia ley para supervisar el cumplimiento efectivo de la misma, el Consejo de Transparencia y Buen Gobierno. El preámbulo de la ley lo define como "un órgano independiente al que se le otorgan competencias de promoción de la cultura de transparencia en la actividad de la Administración Pública, de control del cumplimiento de las obligaciones de publicidad activa, 
así como de garantía del derecho de acceso a la información pública y de la observancia de las disposiciones de buen gobierno. Se crea, por lo tanto, un órgano de supervisión y control para garantizar la correcta aplicación de la Ley". Aunque se configure como un órgano independiente con competencias relacionadas con la promoción de la cultura de la transparencia en las actividades realizadas por las administraciones públicas, con el control del cumplimiento de las obligaciones de publicidad activa y para asegurar el derecho de acceso a la información pública, por su sistema de elección y ubicación se prevén dudas como órgano efectivo de supervisión y control de la aplicación de la ley.

El Consejo de Transparencia y Buen Gobierno dispone de un estatuto, con el Real Decreto 919/2014 (España 2014), configurándose como el máximo órgano que debe actuar con independencia y autonomía para la fiscalización de la transparencia y tramitar las reclamaciones de los ciudadanos. Sin embargo, una serie de características de este organismo plantean serias dudas sobre su autonomía e independencia a la hora de actuar:

a) El Consejo está adscrito al Ministerio de Hacienda y Administraciones Públicas, y se crea un vínculo muy estrecho con dicho ministerio al establecer que debe contar con su personal, dado que, como indica el Real Decreto, la creación y puesta en funcionamiento del Consejo de Transparencia y Buen Gobierno no podrá suponer incremento de dotaciones, retribuciones, u otros gastos de personal ni, por otros conceptos, incremento neto de estructura o de personal al servicio del sector público estatal, señalando además que el Consejo se dotará exclusivamente mediante la redistribución de efectivos del Ministerio de Hacienda y Administraciones Públicas, sus organismos y entidades públicas, y su funcionamiento se atenderá con los medios materiales y personales de que dispone actualmente la Administración.

b) La forma de elección del Presidente del Consejo de Transparencia y Buen Gobierno, que es nombrado por real decreto del Consejo de Ministros a propuesta del titular del Ministerio de Hacienda y Administraciones Públicas. Como trámite protocolario/parlamentario, el candidato propuesto por el Ministerio debe comparecer ante la comisión competente del Congreso de los Diputados que, por acuerdo adoptado por mayoría absoluta, deberá refrendar la propuesta de nombramiento. 
La Ley 19/2013 no especifica nada sobre que se realicen auditorías en torno a la gestión de la transparencia en las administraciones. Simplemente especifica que la Administración General del Estado será objeto de control por parte del Consejo de Transparencia y Buen Gobierno, y en sus funciones no se contempla la fiscalización o auditoría, que se limita a: adoptar recomendaciones; asesorar en materia de transparencia y acceso a la información pública; evaluar el grado de aplicación de la Ley; promover la elaboración de borradores de recomendaciones y de directrices y normas de desarrollo de buenas prácticas en materia de transparencia, acceso a la información pública y buen gobierno; promover actividades de formación y sensibilización; o colaborar, en las materias que le son propias, con órganos de naturaleza análoga.

En el caso de la Ley 19/2014, de 29 de diciembre, de transparencia, acceso a la información pública y buen gobierno de Cataluña (Cataluña 2014), se crea el órgano denominado Comisión de Garantía del Derecho de Acceso a la Información Pública, que debe cumplir sus funciones con plena independencia, compuesta entre tres y cinco miembros, designados por las tres quintas partes de los diputados del Parlamento de Cataluña. En este caso sí que se especifica el perfil profesional de los componentes de la Comisión: juristas especialistas en derecho público y técnicos en materia de archivos o gestión documental, elegidos entre expertos de reconocida competencia y prestigio y con más de diez años de experiencia profesional (art. 40.3). No existe una vinculación tan directa entre el órgano que debe vigilar la transparencia y la propia administración como en la ley estatal. Pero, igualmente, no contempla la posibilidad de realización de auditorías para la fiscalización directa del cumplimiento de la ley. Esta ley contemplaba una creación de registro de grupos de interés que ha sido derogado.

En el procedimiento sancionador de la Ley 19/2013 de Transparencia se indica que "se iniciará de oficio, por acuerdo del órgano competente, bien por propia iniciativa o como consecuencia de orden superior, petición razonada de otros órganos o denuncia de los ciudadanos" con la posibilidad de dar conocimiento de los hechos al Tribunal de Cuentas, por si procediese la incoación de un procedimiento de responsabilidad contable. Es la opción más próxima a la idea de una auditoría por motivos de transparencia, pero, no siendo ese procedimiento, permite que este órgano ajeno a la transparencia realice las averiguaciones oportunas sobre la gestión económica local. La Intervención General de la Administración del Estado ha regulado mediante unas Normas 
de Auditoría (España 1998) como interviene en el sector público, pero el Consejo de Transparencia y Buen Gobierno no puede abordar este asunto porque no lo contempla la legislación mencionada.

\section{Conclusiones}

La transparencia en el sector público es relevante más allá de poner a disposición de los ciudadanos, por Internet (transparencia activa), una cantidad limitada de información producida por las administraciones públicas. La importancia de la transparencia reside en generar confianza de los ciudadanos respecto a las actividades que realizan sus gobernantes o representantes políticos.

Los órganos creados para asegurar el buen cumplimiento de la transparencia no han contemplado la posibilidad de realizar auditorías o fiscalizaciones que den fe del correcto desarrollo de la ley de transparencia. Los órganos auditores regulados por el Estado (Tribunal de Cuentas, Síndic de Comptes, etc.) se centran fundamentalmente en la correcta administración de las cuentas. Una auditoría independiente incorporada a la gestión de la transparencia aseguraría la excelencia en el cumplimiento de la normativa jurídica y potenciaría el grado de confianza de los ciudadanos en el sector público.

\section{Bibliografía}

CARRIEDO, Luis Miguel, 2007. Periodismo y transparencia. Tercer Encuentro de Organizaciones Civiles [en línea]. México. [Consulta: 12/09/2016]. Disponible en: http://www.derechoasaber.org.mx/comunidades/boletines $\% 20 \mathrm{y} \% 20$ ponenc ias/Periodismo y transparencia.pdf

CATALUÑA, 2014. Ley 19/2014, de 29 de diciembre, de transparencia, acceso a la información pública y buen gobierno. BOE [en línea], núm. 18, de 21 de enero de 2015 [Consulta: 22/10/2017]. Disponible en: https://www.boe.es/buscar/pdf/2015/BOE-A-2015-470-consolidado.pdf

ESPAÑA, 1998. Resolución de 1 de septiembre de 1998, de la Intervención General de la Administración del Estado, por la que se ordena la publicación de la Resolución que aprueba las Normas de Auditoría del Sector Público. BOE [en línea], núm. 234, de 30 de septiembre de 1998, pp. 32568-32590 [Consulta: 2/12/2017]. Disponible en: https://www.boe.es/buscar/doc.php?id=BOE$\underline{\text { A-1998-22640 }}$ 
ESPAÑA, 2013. Ley 19/2013, de 9 de diciembre, de transparencia, acceso a la información pública y buen gobierno. BOE [en línea], núm. 295, de 10/12/2013 [Consulta: 20/10/2017]. Disponible en: https://www.boe.es/buscar/act.php?id=BOE-A-2013-12887

ESPAÑA, 2014. Real Decreto 919/2014, de 31 de octubre, por el que se aprueba el Estatuto del Consejo de Transparencia y Buen Gobierno. BOE [en línea], núm. 268, de 05/11/2014 [Consulta: 21/10/2017]. Disponible en: http://www.boe.es/buscar/act.php?id=BOE-A-2014-11410

FRANKLIN F., BENJAMÍN, Enrique, 2007. Auditoria administrativa: gestión estratégica del cambio. México D.F.: Pearson Educación.

GEGINAT, C.; SALTANE, V., 2016. “Open for Business?” —Transparent government and business regulation. Journal of Economics and Business [en línea], 88, November-December, 1-21. [Consulta: 18/10/2017]. http://dx.doi.org/10.1016/j.jeconbus.2016.06.001

GÉRTRUDIX, Manuel; GERTRUDIS-CASADO, María-Carmen; ÁLVAREZGARCÍA, Sergio, 2016. Consumption of public institutions open data by Spanish citizens. El profesional de la información [en línea], 25(4), 535-544. [Consulta: 17/10/2017]DOI http://dx.doi.org/10.3145/epi.2016.jul.03

GIMÉNEZ-CHORNET, Vicent, 2012. Acceso de los ciudadanos a los documentos como transparencia de la gestión pública. Elprofesional de la información [en línea], septiembre-octubre, 21(5), pp. 504-508. [Consulta: 17/10/2017] DOI http://dx.doi.org/10.3145/epi.2012.sep.09

GUNNLAUGSDOTTIR, J., 2016. Reasons for the poor provision of information by the government: public opinion. Records Management Journal [en línea], 26(2), 185-205. [Consulta: 16/10/2017] DOI: http://dx.doi.org/10.1108/RMJ-03$\underline{2015-0013}$

LOURENÇO, R.P., 2015. An analysis of open government portals: A perspective of transparency for accountability. Government Information Quarterly [en línea], 32(3), 323-332. [Consulta: 21/10/2017] DOI: http://dx.doi.org/10.1016/i.giq.2015.05.006

MAYERNIK, M.S.; PHILLIPS, J.; NIENHOUSE, E., 2016. Linking publications and data: Challenges, trends, and opportunities. D-Lib Magazine [en línea], 22(5-6), 1-1. [Consulta: 01/11/2017] DOI: 10.1045/may2016-mayernik

MCNUTT, J.G.; JUSTICE, J.B.; MELITSKI, J.M.; AHN, M.J.; SIDDIQUI, S.R.; CARTER, D.T.; KLINE, A.D., 2016. The diffusion of civic technology and open government in the United States. Information Polity [en línea], 21(2), 153170. [Consulta: 29/10/2017] DOI: 10.3233/IP-160385

OKAMOTO, K., 2016. What is being done with open government data? An exploratory analysis of public uses of New York City open data. Webology [en línea], 13(1), 12p. [Consulta: 28/10/2017] Disponible en: http://www.webology.org/2016/v13n1/a142.pdf

OZTOPRAK, A.A.; RUIJER, E., 2016. Variants of transparency: an analysis of the English Local Government Transparency Code 2015. Local Government Studies 
[en línea], 42(4), 536-556. [Consulta: 1/11/2017] DOI: $10.1080 / 03003930.2016 .1154845$

SÁ, C.; GRIECO, J., 2016. Open Data for Science, Policy, and the Public Good. Review of Policy Research [en línea], 33 (5), 526-543. [Consulta: 27/10/2017] doi:10.1111/ropr.12188

TRANSPARENCIA INTERNACIONAL, 2017. Qué es Transparencia Internacional [en línea]. [Consulta: 18/10/2017]. Disponible en: http://transparencia.org.es/que-es-ti/

VAN LOENEN, B.; Kulk, S.; PLOEGER, H., 2016. Data protection legislation: A very hungry caterpillar. The case of mapping data in the European Union. Government Information Quarterly [en línea], 33(2), 338-345. [Consulta: 2/11/2017] http://dx.doi.org/10.1016/j.giq.2016.04.002

VETRÒ, A.; CANOVA, L.; TORCHIANO, M.; MINOTAS, C.O.; IEMMA, R.; MORANDO, F., 2016. Open data quality measurement framework: Definition and application to Open Government Data. Government Information Quarterly [en línea], 33(2), 325-337. [Consulta: 28/10/2017] http://dx.doi.org/10.1016/j.giq.2016.02.001

WANG, MC., LEE, MH. \& CHUANG, JJ., 2016. Relations among audit committee establishment, information transparency and earnings quality: evidence from simultaneous equation models. Quality \& Quantity [en línea], 50: 2417. [Consulta: 28/10/2017] doi:10.1007/s11135-015-0269-y

YAQOOB, I.; HASHEM, I.A.T.; GANI, A.; MOKHTAR, S.; AHMED, E.; ANUAR, N.B.; VASILAKOS, A.V., 2016. Big data: From beginning to future. International Journal of Information Management [en línea], 36(6), 1231-1247. [Consulta: 26/10/2017] http://dx.doi.org/10.1016/j.ijinfomgt.2016.07.009 